\title{
Involvement of GluA1-AMPAR-mediated LTP in time-dependent decline of cognitive function in rats with temporal lobe epilepsy
}

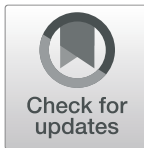

Xiaoqing Luo ${ }^{\dagger}$, Xiaoli Yư ${ }^{\dagger}$, Jufang Liang, Ruidi Sun, Cheng Li and Jun Jiang ${ }^{*}$

\begin{abstract}
Background: Cognitive impairment is one of the common comorbidities in patients with temporal lobe epilepsy (TLE), but the underlying mechanisms remain largely unknown. Previous studies have found significant decay of hippocampal long-term potentiation (LTP) in TLE rats with cognitive impairment. As the activation of a-amino-3hydroxy-5-methyl-4-isoxazole propionic acid receptors (AMPARs) is responsible for LTP formation and learning and memory, we investigated whether AMPARs are involved in the LTP inhibition and the TLE-associated cognitive impairments.

Methods: TLE rat model was established by intraperitoneal injection of lithium chloride-pilocarpine on postnatal day 21 (P21). Learning and memory performance, hippocampal expression of membrane GluA1-AMPARs, and hippocampal LTP were tested by behavioral tests, western blotting, and field potential recording, respectively, at 1 , 5 and 13 weeks after induction of status epilepticu (SE). Finally, the effects of (S)-AMPA, an agonist of AMPARs, on LTP and cognitive function were tested.

Results: Results of behavioral tests revealed an time-dependent decline in the learning and memory of TLE rats when compared to the age-matched controls at week 5 and 13, rather than at week 1 after the induction of SE. Western blotting showed that the hippocampal expression of membrane GluA1 was significantly decreased in a time-dependent manner in the TLE rats when compared to the age-matched controls at weeks 5 and 13, rather than at week 1 after the induction of SE. Similarly, the hippocampal LTP was inhibited in a time-dependent manner in TLE rats at weeks 5 and 13, rather than at week 1 after the induction of SE. Moreover, intra-hippocampal injection of (S)-AMPA ameliorated the deficits in learning as well as spatial and emotional memory in a dosedependent manner, and partially reversed the inhibition of CA1 LTP in the TLE rats at week 13 after the induction of SE.
\end{abstract}

Conclusions: The reduced expression of hippocampal membrane GluA1 may be involved in LTP decay in CA1 and cognition impairment in TLE rats.

Keywords: Cognitive impairment, Temporal lobe epilepsy, Long-term potentiation, Hippocampus, AMPA receptor

\footnotetext{
* Correspondence: jiangjuntyz@163.com

${ }^{+}$Xiao-Qing Luo and Xiao-Li Yu contributed equally to this work.

Department of Electrophysiology, Wuhan Children's Hospital (Wuhan

Maternal and Child Healthcare Hospital), Tongji Medical College, Huazhong

University of Science and Technology, No.100 of Hong Kong Road, Jiang'an

District, 430016 Wuhan, China
}

(c) The Author(s). 2021 Open Access This article is licensed under a Creative Commons Attribution 4.0 International License, which permits use, sharing, adaptation, distribution and reproduction in any medium or format, as long as you give appropriate credit to the original author(s) and the source, provide a link to the Creative Commons licence, and indicate if changes were made. The images or other third party material in this article are included in the article's Creative Commons licence, unless indicated otherwise in a credit line to the material. If material is not included in the article's Creative Commons licence and your intended use is not permitted by statutory regulation or exceeds the permitted use, you will need to obtain permission directly from the copyright holder. To view a copy of this licence, visit http://creativecommons.org/licenses/by/4.0/. 


\section{Background}

Temporal lobe epilepsy (TLE), characterized by recurrent and spontaneous seizures, is the most common type of adult epilepsy [1]. About 30\% of TLE patients suffer from certain degrees of cognitive impairment such as learning and memory deficits, which can cause even greater impacts on the quality of life than seizures per se [2-4]. As the underlying causes of cognitive impairment in TLE are not clear, current treatment options for epilepsy-associated cognitive impairment are limited. Several recent studies have emphasized the importance of studying the age-related aspects of neurocognitive impairment in epileptic patients for a better understanding of the pathogenesis of comorbidities [5-7]. However, it is unclear whether the cognitive function in TLE gets worse over time.

The hippocampus plays a vital role in spatial navigation, episodic memory and long-term memory [8, 9], and is vulnerable to hypoxic, ischemic, and neurotoxic insults. Therefore, the hippocampus is also involved in pathophysiological mechanisms of various neurological disorders such as epilepsy and cognitive impairment [10]. Several lines of evidence have suggested that recurrent seizures can lead to qualitative changes of hippocampal neurogenesis, which are associated with cognitive impairment in the developing and mature brains [11]. Long-term potentiation (LTP) is the most widely studied manifestation of synaptic plasticity and is a physiological model of learning and memory [12]. Our previous studies showed that the LTP decay in hippocampal CA1 region may be one of the contributors to the cognitive dysfunction in TLE rats induced by lithium chloride-pilocarpine [13]. However, the underlying mechanisms of LTP decay and its correlation with age are not well understood.

Alpha-amino-3-hydroxy-5-methyl-4-isoxazole propionic acid receptors (AMPARs) are the major glutamate receptor mediating fast synaptic transmission in the brain. Numerous studies have shown that AMPARs are fundamental in LTP maintenance and hippocampal learning and memory [14]. AMPARs are cation-selective tetrameric hetero-oligomers composed of four subunits, GluA1-4 [15]. Accumulating evidence has suggested that AMPARs undergo constant dynamic remodeling in response to pathological conditions [16-18]. A strong body of evidence has suggested that the hippocampal levels of membrane GluA1 are essential for learning and memory. Besides, the hippocampal trafficking of GluA1containing AMPARs could affect cognitive behaviors [19-21]. It has been reported that mice lacking the GluA1 subunit present LTP inhibition and hippocampal-dependent spatial memory impairment[21]. However, it is unclear whether GluA1 is involved in the hippocampal LTP suppression in TLE rats.
In this study, we set out to examine the correlation between cognitive impairment and age in TLE rats, and explore whether the AMPARs are involved in LTP and TLE-associated cognitive impairment.

\section{Materials and Methods}

Rat model of epilepsy

Male Sprague-Dawley rats aged 21 days were taken from the Experimental Animal Center of Tongji Medical College, Huazhong University of Science and Technology. The rats were given $3 \mathrm{mg} / \mathrm{kg}$ lithium chloride (i.p.), followed $\sim 20 \mathrm{~h}$ later by $25 \mathrm{mg} / \mathrm{kg}$ pilocarpine (i.p.). The rats were given an i.p. injection of $1 \mathrm{mg} / \mathrm{kg}$ atropinemethyl nitrate $30 \mathrm{~min}$ before pilocarpine was given. Status epilepticus (SE) was defined according to Racine score. Then convulsions $60 \mathrm{~min}$ after SE was terminated by $10 \%$ chloraldurate $(3 \mathrm{~mL} / \mathrm{kg}$, i.p.).

\section{Morris water maze test}

The Morris water maze, a 50-cm high circular tank with a diameter of $100 \mathrm{~cm}$ was filled with water $\left(25^{\circ} \mathrm{C}\right)$ and a platform was placed $2 \mathrm{~cm}$ below the surface of water. When the rats found the platform, they were allowed to stay on it for 20 seconds. Rats that could not find the platform were guided to the platform and stayed there for 20 seconds. The platform was removed one day after the last training day. In the test session, the escape latency and distance of rats to find the hidden platform was recorded to examine the spatial long-term memory. Time spent in target quadrant within 120 seconds were recorded.

\section{Passive avoidance test}

The passive avoidance test was conducted in a closed and quiet device, which consisted of a dark compartment with an electric foot-shock and a light compartment separated by a guillotine door. The rats were placed in the light compartment, and allowed to enter the dark compartment after the guillotine door was open 10 seconds later. Once a rat has fully entered the dark compartment, the guillotine door is immediately closed, and the grid floor delivers an electric foot-shock $(0.5 \mathrm{~mA}, 2 \mathrm{~s})$. On the next day, the rat was again placed in the light compartment, and 10 seconds later the guillotine door opened. The latency to enter the dark compartment was recorded.

\section{Hippocampal slice preparation and electrophysiological recordings}

Hippocampal LTP was tested by field potential recording in vitro as described in our previous study [22]. Briefly, the rats were sacrificed by decapitation under intraperitoneal anesthesia and the rat brain was immediately placed in ice-cold artificial cerebrospinal fluid bubbled 
with $95 \% \mathrm{O}_{2}$ and $5 \% \mathrm{CO}_{2}$. As reported in our previous experiments, the field excitatory postsynaptic potentials (fEPSPs) were recorded from the dendritic layer of CA1 pyramidal cells [22].

\section{Hippocampal membrane protein fractionation and Western blotting analysis}

Briefly, the hippocampal tissues were gathered, resuspended and homogenized. The post-synaptic density (PSD) pellet was obtained by centrifugation, lysis, and sucrose gradient. The hippocampal membrane proteins from the PSD pellet were extracted using the ProteoExtract Transmembrane Protein Extraction Kit. Proteins were probed with primary mouse anti- $\beta$-actin monoclonal antibody (1:1 000; EarthOx Life Sciences,USA, Cat\# E021020, RRID: AB_2572416) and antibodies rabbit anti-GluA1 polyclonal antibody (1:1 000; Millipore, USA, Cat\#AB1504, RRID: AB_2113602) at $4{ }^{\circ} \mathrm{C}$ overnight, and incubated with the horseradish peroxidaselinked goat anti-mouse IgG (1:5 000, Abcam, UK) for $1 \mathrm{~h}$ on the following day.

\section{Surgery and intra-hippocampal injection}

The rat was placed on a stereotaxic instrument after anesthetization, and the skull was fully exposed. Two guide cannulas were placed stereotaxically and directed into the CA1 region of the hippocampus according to our previous experiments [22]. A stainless-steel injection tube was directly inserted into the guide cannula after 3day recovery from the operation. Then $2 \mu \mathrm{l}$ of $0.1 \mu \mathrm{g} / \mu \mathrm{l}$ (S)-AMPA was injected into the hippocampal CA1 region within $5 \mathrm{~min}$ and was left in place for an additional 5 min to allow diffusion in TLE rats at week 13 after SE induction.

\section{Statistical analysis}

All data are expressed as mean \pm SEM and analyzed with the SPSS 10.0 software. The difference in learning and memory function between control and TLE rats was analyzed by one-way repeated measures analysis of variance (ANOVA) followed by Turkey's HSD post-hoc test. Hippocampal field potential was analyzed by two-way ANOVA. Western blot results were analyzed using unpaired $t$-test. A $p<0.05$ was considered statistically significant.

\section{Results}

Time-dependent decline of cognitive function in TLE rats Animals with Racine scores of 4-5 were used in the following experiments. Morris water maze showed no significant differences in learning and memory between TLE rats and controls at week 1, 5 and 13 after induction of SE (Fig. 1). There were significant differences in the escape latency and the mean distance traveled on day 4 and 5 of training between TLE rats and controls at week 5 after induction of SE (ANOVA, $p<0.05$, Fig. 1b, e). At week 13, the learning and memory ability of the TLE rats was significantly impaired as compared to the controls (ANOVA, $p<0.05$, Fig. 1c, f). Meanwhile, the escape latency and the mean distance traveled of TLE rats were significantly increased at week 13 compared to the those at week 5 . In addition, the time spent in the target quadrant on day 6 was significantly reduced compared to the controls at weeks 5 and 13 (ANOVA, $p<0.05$, Fig. 1g). Meanwhile, the time TLE rats spent in the target quadrant significantly differed between weeks 5 and 13 after SE induction (ANOVA, $p<0.05$, Fig. 1 g). In the passive avoidance test, there were significant differences in the step-through latency between TLE rats and control rats at weeks 5 and 13 after SE induction (ANOVA, $p<0.05$, Fig. 1h). Meanwhile, the TLE rats spent more time to enter the dark compartment at week 13 than that at week 5 ( $p<0.05$, Fig. $1 \mathrm{~h})$. These results indicated time-dependent spatial memory defects in the TLE rats.

\section{Time-dependent inhibition of hippocampal LTP in TLE rats}

There were no significant differences in the slope and amplitude of hippocampal CA1 fEPSPs in control rats among weeks 1, 5, and 13 after SE induction (Fig. 2a, c). The hippocampal LTP at week 1 after SE induction did not differ between TLE and control rats. The LTP slope of TLE rats was decreased by $27.77 \%$ and $59.58 \%$ at weeks 5 and 13, respectively, as compared to that of the controls, and the amplitude was reduced by $35.43 \%$ and $51.37 \%$, respectively. The LTP slope and amplitude of TLE rats were decreased by $29.22 \%$ and $28.02 \%$ at week 5 , respectively, when compared those at week 1 . The LTP slope and amplitude of TLE rats were decreased by $63.86 \%$ and $50.03 \%$ at week 13 , respectively, when compared those at week 1 . These results implied that the LTP of CA1 hippocampal was time-dependently inhibited in TLE rats.

\section{Time-dependent decrease of hippocampal GluA1 protein in TLE rats}

Results of Western blotting showed that the protein level of hippocampal membrane GluA1 in the TLE rats did not change at week 1 after SE induction, but was decreased by $49.83 \%$ and $72.18 \%$, respectively, at weeks 5 and 13 as compared to the age-matched controls (unpaired t-test, $p<0.05$, Fig. 3). In addition, the protein level of hippocampal membrane GluA1 in the TLE rats was decreased by $47.53 \%$ at week 5 compared to that at week 1 after SE induction (unpaired $t$-test, $p<0.05$, Fig. 3), and was decreased by $50.98 \%$ at week 13 compared to that at week 5 after SE induction (unpaired ttest, $p<0.05$, Fig. 3 ). These results implied that the 


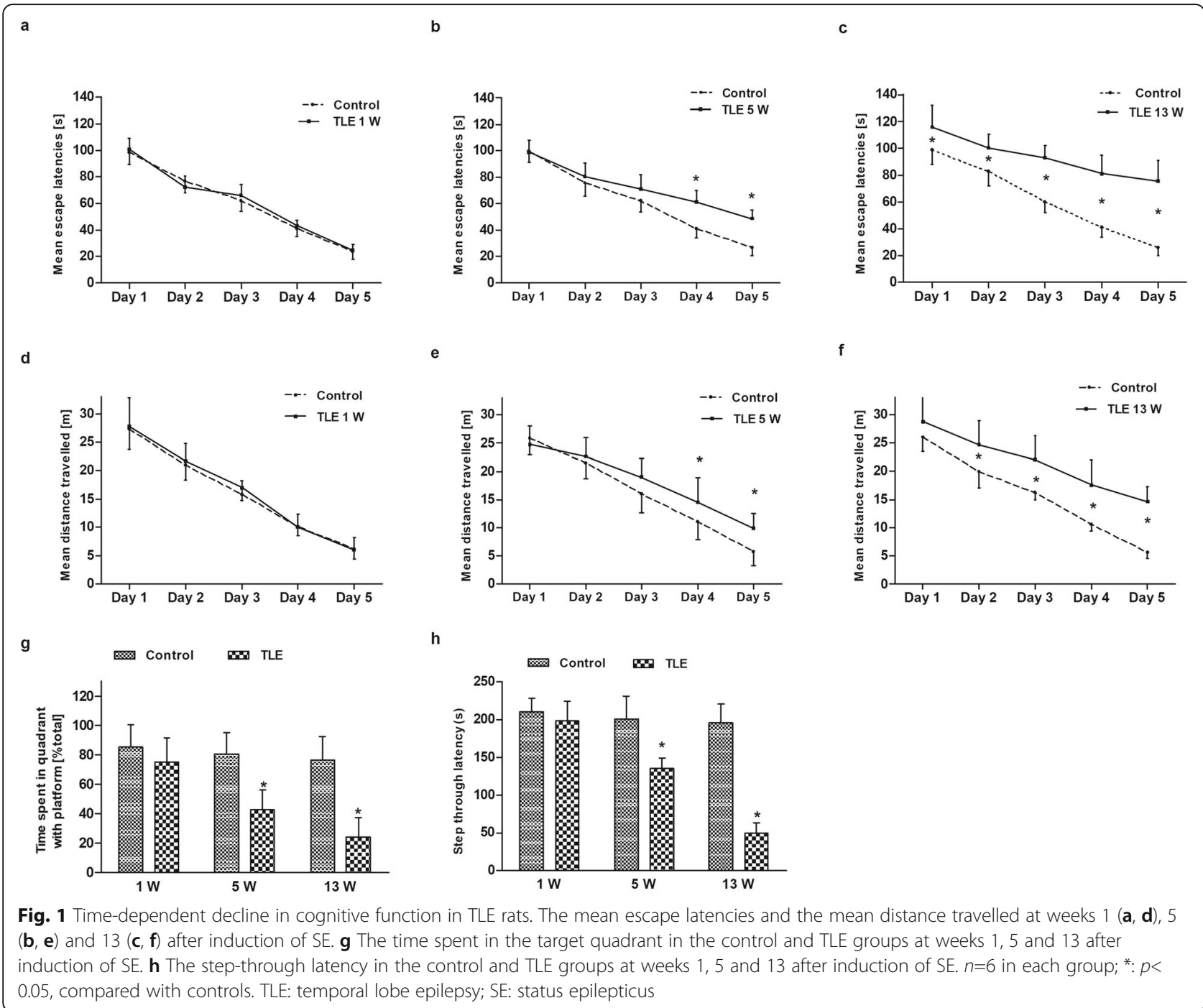

hippocampal membrane GluA1 was decreased in a timedependent manner, and may be correlated with the timedependent decline of cognitive function in the TLE rats.

\section{AMPARs agonist improved learning and memory ability of TLE rats}

TLE rats at week 13 after SE induction were used to detect the influence of AMPARs in learning and memory ability. Treatment of $0.1 \mu \mathrm{g} / \mu \mathrm{l}(S)$-AMPA did not significantly alter the escape latency, the time spent in the target quadrant, and the step-through latency of the TLE (Fig. 4a-c). The escape latency of the TLE rats was decreased by $10.64 \%, 14.98 \%$ and $28.56 \%$ by $0.4 \mu \mathrm{g} / \mu \mathrm{l}(S)$-AMPA on day 3,4 and 5 of that week as compared to the $0.1 \mu \mathrm{g} / \mu \mathrm{l}(S)$-AMPA group (ANOVA, $p<0.05$, Fig. 4a). The time spent in the target quadrant was increased by $25.88 \%$ after injection of $0.4 \mu \mathrm{g} / \mu \mathrm{l}(S)$-AMPA compared with $0.1 \mu \mathrm{g} / \mu \mathrm{l}(S)$-AMPA group (ANOVA, $p<0.05$, Fig. $4 \mathrm{~b}$ ). The step-through latency was increased by $31.52 \%$ after injection of
$0.4 \mu \mathrm{g} / \mu \mathrm{l}(S)$-AMPA compared with the $0.1 \mu \mathrm{g} / \mu \mathrm{l}(S)$-AMPA group (ANOVA, $p<0.05$, Fig. 4c). The escape latency was decreased by $15.48 \%, 27.90 \%$ and $32.35 \%$ on day 3,4 and 5 , respectively, after injection of $0.8 \mu \mathrm{g} / \mu \mathrm{l}(S)$-AMPA, compared to the $0.4 \mu \mathrm{g} / \mu \mathrm{l}(S)$-AMPA group (ANOVA, $p<0.05$, Fig. 4a). The time spent in the target quadrant was increased by $35.73 \%$ after injection of $0.8 \mu \mathrm{g} / \mu \mathrm{l}(S)$-AMPA compared to the $0.4 \mu \mathrm{g} / \mu \mathrm{l}(S)$ AMPA group (ANOVA, $p<0.05$, Fig. $4 \mathrm{~b}$ ). The step-through latency was increased by $33.94 \%$ after injection of $0.8 \mu \mathrm{g} / \mu \mathrm{l}(S)$ AMPA compared to the $0.4 \mu \mathrm{g} / \mu \mathrm{l}(S)$-AMPA group (ANOVA, $p<0.05$, Fig. 4c). These data demonstrated that the intrahippocampal injection of the AMPARs agonist ameliorated the cognitive impairment of TLE rats in a dose-dependent manner.

The potentiation effects of (S)-AMPA on hippocampal LTP in TLE rats

As shown in Fig. 5, 2.0 $\mu \mathrm{M}$ of (S)-AMPA enhanced the amplitude and slope of fEPSPs of by hippocampal CA1 

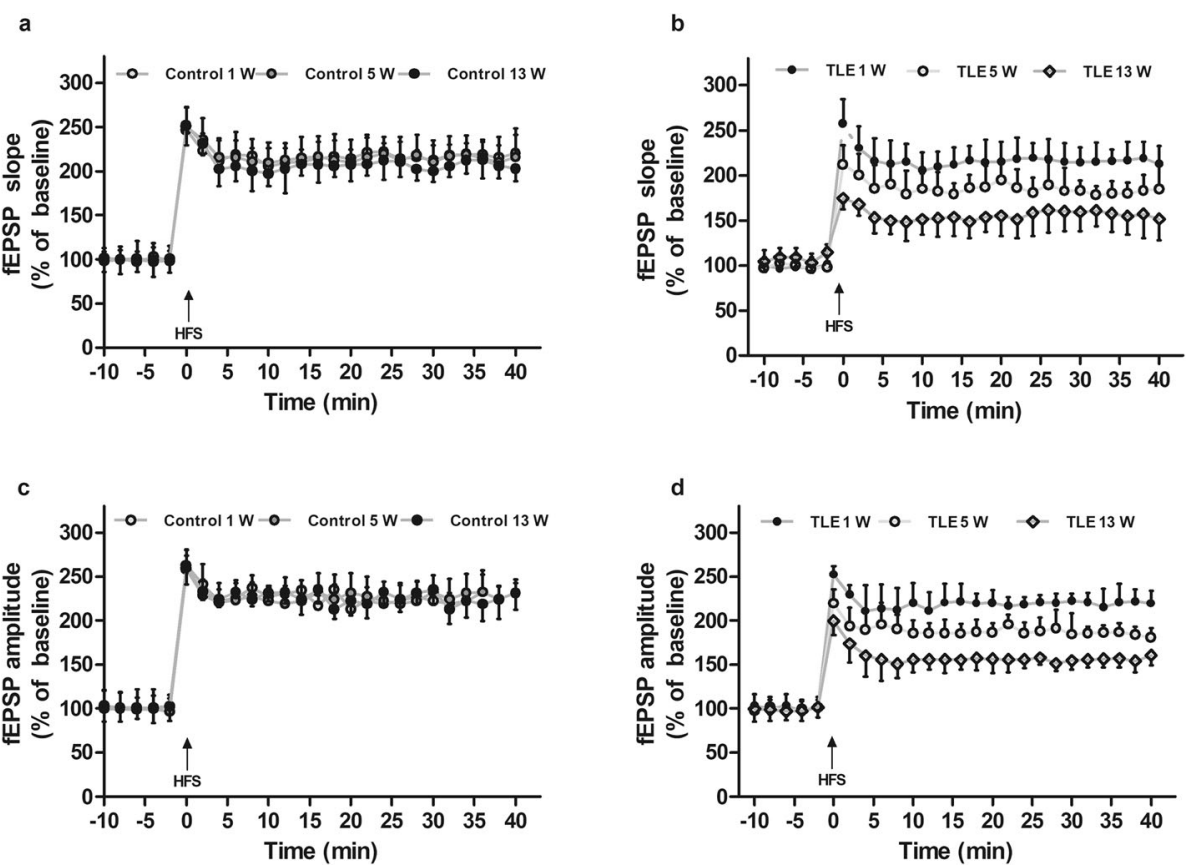

Fig. 2 Time-dependent inhibition of hippocampal LTP in TLE rats.The standardized fEPSPs slope and amplitude in control (a, c) and TLE rats (b, d) at weeks 1, 5 and 13 after induction of SE. $n=8$ slices from four rats in each group. LTP: long-term potentiation; TLE: temporal lobe epilepsy; fEPSP: field excitatory postsynaptic potentials; HFS: high frequency stimulation

LTP 24.83\% (range 154.42-192.78\%) and 24.53\% (range 154.71-192.67\%), respectively, at $40 \mathrm{~min}$ after high frequency stimulation (HFS) in TLE rats at week 13 after SE induction as compared to the saline group $(p<0.05$, Fig. $5 \mathrm{a}$ and $\mathrm{b})$, suggesting that the inhibition of CA1 hippocampal LTP in TLE rats was partially reversed by $(S)$ AMPA. These results presented that AMPARs may play an important role in the LTP decay of the hippocampal CA1 region from TLE rats with cognitive impairment.

\section{Discussion}

In this study, we revealed time-dependent impairment in learning and memory of TLE rats and demonstrated that the decreased hippocampal membrane GluA1 may be involved in the inhibition of CA1 LTP, which may underlie the time-dependent cognitive impairment of the TLE rats.

\section{Time-dependent impairment of learning and memory in the TLE rats}

There is compelling evidence that uncontrolled seizures and interictal spikes contribute to cognitive deterioration over time [6]. The age-related aspects have received more attention in recent studies of epileptic comorbidities in the aim to better understand the comorbidities of epilepsy. Hermann et al. have reported that neurobehavioral comorbidities during seizures are the main signs of cognitive dysplasia before and after seizures [5]. Helmstaedter and Elger suggested that early control of epilepsy is required to offset developmental disorders and damage at a younger age to improve cognitive impairment [4]. Karson et al. reported that the WAG/Rij rat model of absence epilepsy presented time-dependent learning and memory deficits [7]. Consistently, our study revealed that TLE rats exhibited behavioral manifestations of cognitive impairment in early time of epilepsy and time-dependent memory deficits. There is also a considerable amount of evidence indicating that the mechanisms of pathological brain aging might also be implicated in the cognitive dysfunction in epilepsy patients, such as the age-accelerated ventricular expansion which is characteristic of advanced brain aging and the increased amyloid- $\beta 42$ burden in chronic and refractory epilepsy [23-25]. In our experiment, the agematched control rats were tested to eliminate the disturbing influence of age on cognitive function. However, it remains to be clarified whether chronic epilepsy would accelerate the process of brain aging and result in cognitive impairment in TLE.

\section{The effects of hippocampal LTP decay on cognitive impairment}

The hippocampal CA1 LTP is closely associated with learning and memory. Previous studies have reported that seizures, including kindling, can result in a shortterm increase of hippocampal CA1 synaptic strength $[11,26]$. On the other hand, several lines of evidence have suggested the occurrence of reduction or 


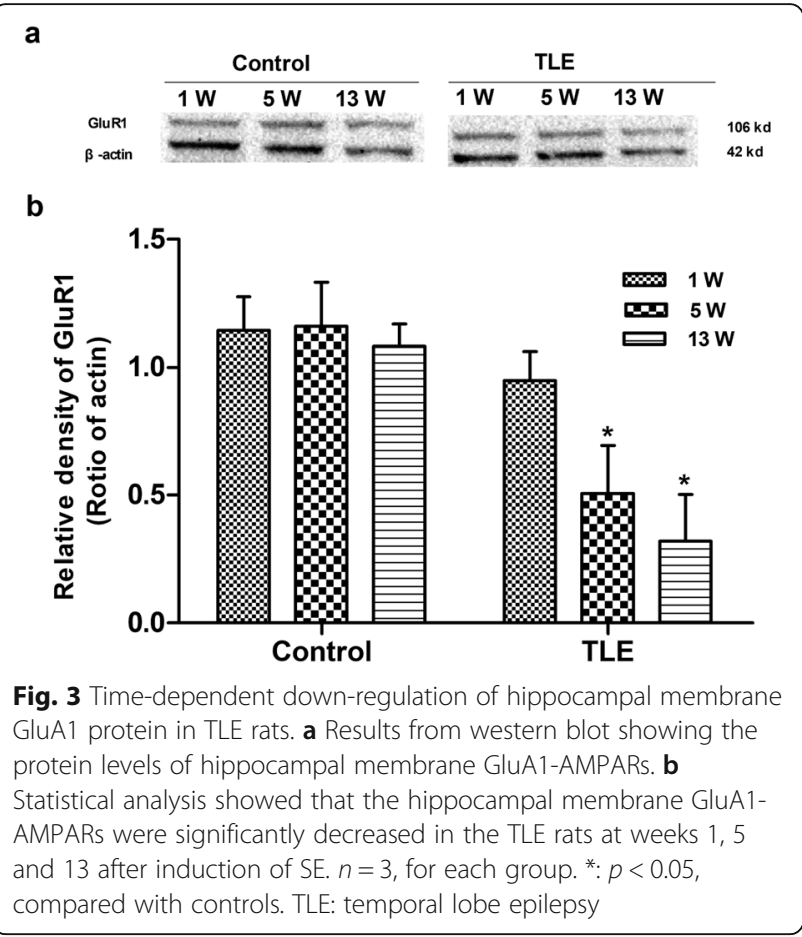

elimination of LTP at days to weeks after SE, which contributes to the learning and memory deficits [12]. In our previous experiments, the TLE rats showed hippocampal CA1 LTP decay in vivo, suggestive of deficits of learning and memory. In our study, the (S)-AMPA partly reversed the inhibition of CA1 LTP in TLE rats, which implied that the CA1 LTP decay may be involved in cognitive impairment in TLE rats. Zhang et al. reported that the LTP induction was strengthened 1 day after SE induction, returned to normal levels 2 weeks later, and was weakened after 6 weeks [12]. They therefore proposed that there are two stages of synaptic plasticity change after SE induction, the early stage of LTP enhancement and the late stage of LTP reduction. In our study, the LTP of TLE rats was at the normal level at week 1 , but showed a time-dependent decay at weeks 5 and 13 after SE induction. Our findings, together with those of Zhang et al., provide convincing evidence that the time-dependent inhibition of hippocampal LTP may be involved in the impairment of learning and memory ability in epileptic rats.

\section{The effects of GluA1-AMPARs in LTP decay and cognitive impairment}

Numerous lines of evidence have indicated that AMPARs, which are composed of four different subunits GluA1-4, are vital for rapid synaptic transmission, synaptic plasticity of hippocampal neurons and behavioral expression [16]. The GluA1/A2 heteromers are the dominant AMPARs at hippocampal CA1 neuronal synapses. The GluA2-lacking AMPARs are $\mathrm{Ca}^{2+}$-permeable, while the GluR2-AMPARs are $\mathrm{Ca}^{2+}$-impermeable. It has been reported that addition of AMPARs containing GluA1, GluA3 and GluA4 subunits may help maintain the cognitive ability of 3XTG-AD mice before symptoms appear[17]. Ding et al. have reported that the decreased expression and phosphorylation of hippocampal post-synaptic membrane GluA1- containing AMPARs may be related to the impaired cognitive
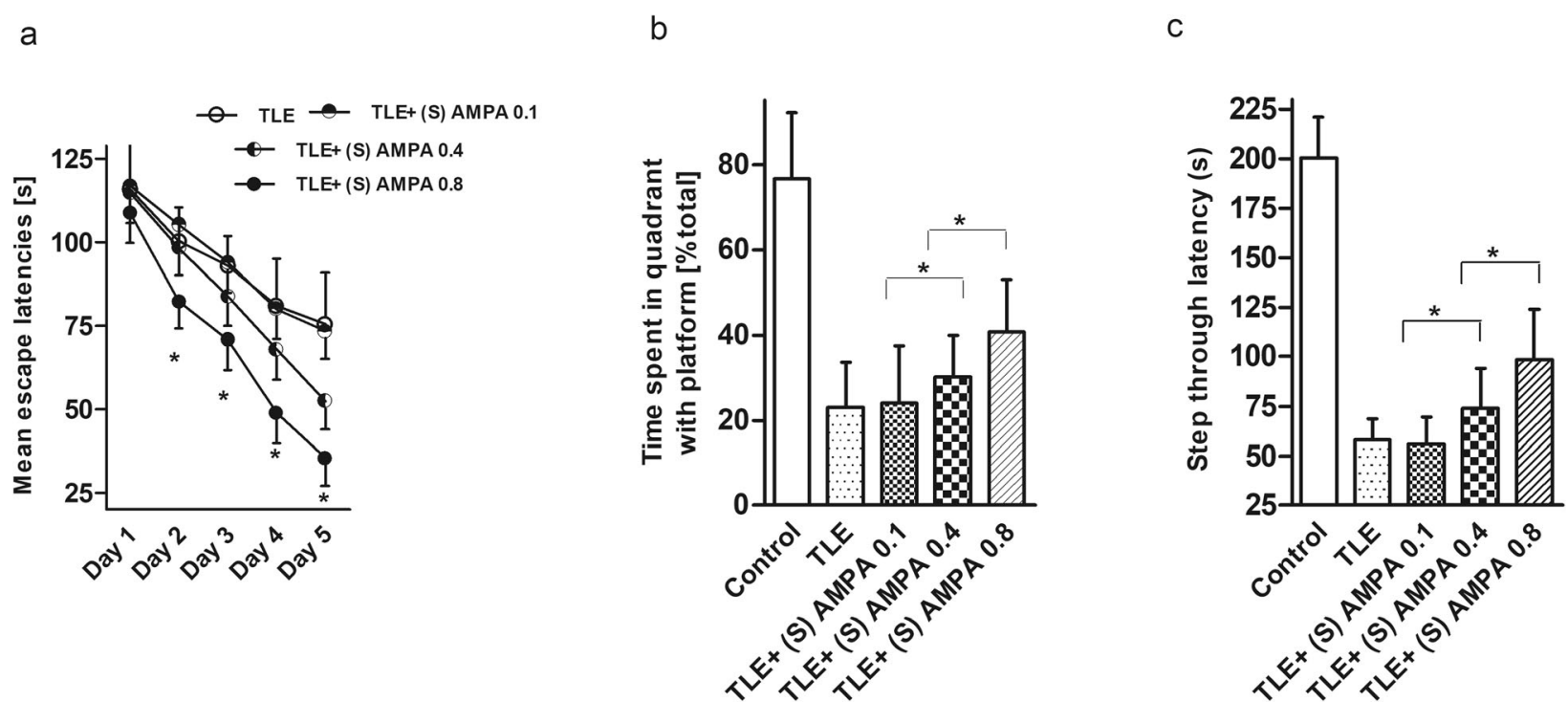

Fig. 4 AMPA receptor agonist improved learning and memory in TLE rats. Mean escape latencies (a), time spent in the quadrant with platform (b), and step-through latency (c) after bilateral intra-hippocampal injection of saline or (S)-AMPA in the TLE rats at week 13 after induction of SE. $n=6$ for each group; *: $p<0.05$, compared with controls. TLE: temporal lobe epilepsy 
a

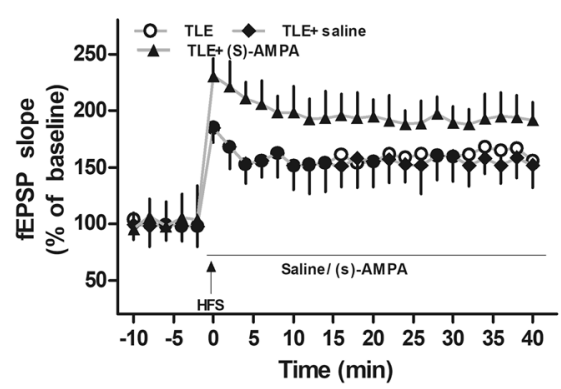

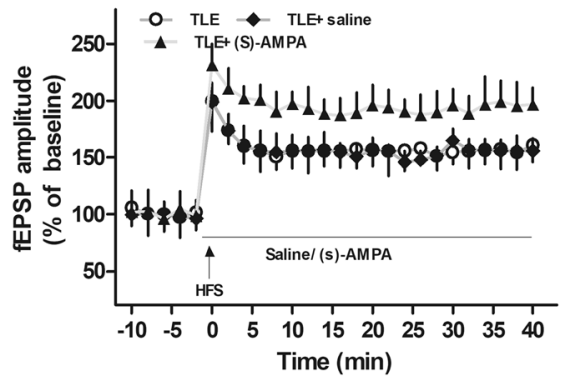

Fig. 5 The potentiation effects of (S)-AMPA on hippocampal LTP in TLE rats. The effect of (S)-AMPA on the standardized fEPSPS slope (a) amplitude (b) in TLE rats. $n=8$ slices from four rats in each group. LTP: long-term potentiation; fEPSPs: field excitatory postsynaptic potentials; TLE: temporal lobe epilepsy

function after long-term ketamine administration [19]. Here, we reported the reduced expression of hippocampal membrane GluA1, which was accompanied by attenuated hippocampal CA1 LTP and deficits of learning and memory ability after SE induction. Moreover, intrahippocampal injection of (S)-AMPA dose-dependently ameliorated the impairment of learning as well as spatial and emotional memory in TLE rats. These findings provide further evidence that the levels of hippocampal membrane GluA1 are fundamental for LTP and cognitive behaviors. An earlier study showed that the GluA1AMPARs levels in each fascia dentata granule neuron in temporal lobe epilepsy were higher than those in the nonseizure autopsies [15]. The different findings among different studies may be explained by the differences in species and strains of animals examined, the time after SE induction, and the tissues tested. On the other hand, several studies have suggested that the GluA2-containing AMPA Rs are involved in cognitive disorders [27, 28]. Therefore, further studies are needed to explore the pathogenesis of cognitive impairment in TLE.

\section{Conclusions}

Our study presented further evidence of learning and memory deficits in the TLE rats, and demonstrated that the reduced expression of hippocampal membrane GluA1 may be involved in CA1 LTP decay and cognition impairment in TLE rats.

\section{Abbreviations}

TLE: Temporal lobe epilepsy; LTP: Long-term potentiation; AMPARs: a-amino3-hydroxy-5-methyl-4-isoxazole propionicacid receptors; i.p.: Intraperitoneal; SE: Status epilepticus; fEPSPs: Field excitatory postsynaptic potentials; HFS: High frequency stimulation

\section{Acknowledgements}

Not applicable.

\section{Authors' contributions}

XQL participated in the generation of animal model and manuscript writing. XLY performed molecular experiments and analyzed the results. JFL carried out animal experiments. RDS participated in the study and analyzed the results. $\mathrm{CL}$ supervised the design of the research. JJ designed and prepared the manuscript. All authors approved the final manuscript.

Funding

This research was supported by the project of Wuhan Municipal Health Planning Commission (WX17Q26/ WX18Y13).

Availability of data and materials

The datasets during and/or analysed during the current study are available from the corresponding author on reasonable request.

Ethics approval and consent to participate

This study was approved by the Animal Care and Use Committee of Huazhong University of Science and Technology.

\section{Consent for publication}

Not applicable.

\section{Competing interests}

The authors (Xiaoqing Luo, Xiaoli Yu, JuFang Liang, Ruidi Sun, Cheng Li and Jun Jiang) declare no competing interests.

Received: 2 November 2020 Accepted: 27 November 2020 Published online: 15 January 2021

\section{References}

1. Lenck-Santini PP, Scott RC. Mechanisms responsible for cognitive impairment in epilepsy. Cold Spring Harb Perspect Med. 2015;5(10):a022772.

2. Helmstaedter C, Witt JA. Epilepsy and cognition-A bidirectional relationship? Seizure. 2017:49:83-9

3. Joplin S, Stewart E, Gascoigne M, Lah S. Memory Rehabilitation in Patients with Epilepsy: a Systematic Review. Neuropsychol Rev. 2018;28(1):88-110.

4. Helmsaedter C, Elger CE, Vogt VL. Cognitive outcomes more than 5 years after temporal lobe epilepsy surgery: Remarkable functional recovery when seizures are controlled. Seizure. 2018:62:116-23.

5. Hermann BP, Jones JE, Sheth R, Koehn M, Becker T, Fine J, et al. Growing up with epilepsy: a two-year investigation of cognitive development in children with new onset epilepsy. Epilepsia. 2018;49:1847-58.

6. Zhang Y, Cai GE, Yang Q, Lu QC, Li ST, Ju G. Time-dependent changes in learning ability and induction of long-term potentiation in the lithiumpilocarpine-induced epileptic mouse model. Epilepsy Behav. 2010;17:448-54

7. Karson A, Utkan T, Balıı F, Arıcıoğlu F, Ateș N. Time-dependent decline in learning and memory performances of WAG/Rij rat model of absence epilepsy. Behav Brain Funct. 2012;8:51.

8. Coras R, Pauli E, Li J, Schwarz M, Rössler K, Buchfelder M, et al. Differential influence of hippocampal subfields to memory formation: insights from patients with temporal lobe epilepsy. Brain. 2014;137:1945-57. 
9. Chen A, Chen Y, Tang Y, Bao CJ, Cui ZZ, Xioa M, et al. Hippocampal AMPA Rs involve the central sensitization of rats with irritablebowel syndrome. Brain Behav. 2017;7:e00650.

10. NakaharaS, Adachi M, Ito H, MatsumotoM, Tajinda K, van Erp TGM HippocampalPathophysiology: Commonality Shared by Temporal Lobe Epilepsyand PsychiatricDisorders. Neurosci J. 2018:4852359.

11. Neumann AR, Raedt R, Steenland HW, Sprengers M, Bzymek K, Navratilova Z, et al. Involvement of fast-spiking cells in ictal sequences during spontaneous seizures in rats with chronic temporal lobe epilepsy. Brain. 2017;140(9):2355-69.

12. Bliss TV, Collingridge GL.A synaptic model of memory: long-term potentiation inthe hippocampus. Nature, 1993, 361 (6407): 31-9.

13. JiangJ, Luo XQ, Li C, Kuang GT, Wang XL, Liang JF, et al. Roles of Hippocampal long-term potentiation inchronic cognitive dysfunction of immature epileptic rats. J Epilepsy. 2016;2(3):220-23.

14. Henley JM. AMPA receptor trafficking and the mechanisms underlying synaptic plasticity and cognitive aging. Dialogues Clin Neurosci. 2013;15:11-27.

15. Mathern GW, Pretorius JK, Kornblum HI, Mendoza D, Lozada A, Leite JP, et al. Human hippocampal AMPA and NMDA mRNA levels in temporal lobe epilepsy patients. Brain. 1997;120:1937-59.

16. Bernard CL. Simultaneous expression of long-term depression of NMDA and long-term potentiation of AMPA receptor-mediated synaptic responses in the CA1 area of the kainic acid-lesioned hippocampus. Eur J Neurosci. 1995; 7:1651-5.

17. Cantanelli P, Sperduti S, Ciavardelli D, Stuppia L, Gatta V, Sensi SL. AgeDependent Modifications of AMPA Receptor Subunit Expression Levels and Related Cognitive Effects in 3xTg-AD Mice. Front Aging Neurosci. 2014;6:200.

18. O'Leary H, Bernard PB, CastanoAM. Enhanced long term potentiation and decreased AMPA receptor desensitizationin the acute period following a single kainate induced early life seizure. NeurobiolDis, 2016, 87: 134-44.

19. Ding R, Li Y, Du A, Yu H, He B, Shen R, et al. Changes in hippocampal AMPA receptors and cognitive impairments in chronic ketamine addiction models: another understanding of ketamine CNS toxicity. Sci Rep. 2016;6:38771.

20. Zhao $L X$, Ge YH, Xiong CH, Tang L, Yan YH, Law PY, et al. M1 muscarinic receptor facilitates cognitive function by interplay with AMPA receptor GluA1 subunit. FASEB J. 2018;32:4247-57.

21. Bannerman DM, Borchardt T, Jensen V, Rozov A, Haj-Yasein NN, Burnashev $\mathrm{N}$, et al. Somatic Accumulation of GluA1-AMPA Receptors Leads to Selective Cognitive Impairments in Mice. Front Mol Neurosci. 2018;11:199.

22. Chen Y, Chen AQ, Luo XQ, Guo LX, Tang Y, Bao C J, et al. Hippocampal NR2B-containingNMDA receptors enhancelong-term Potentiationin rats with chronic visceral pain. Brain Research, 2014;1570:43-53.

23. Helmstaedter $C$. Chronic temporal lobe epilepsy: a neurodevelopmental or progressively dementing disease? Brain. 2009;132:2822-30.

24. Dabbs K, Becker T, Jones J, Rutecki P, Seidenberg M, Hermann B. Brain structure and aging in chronic temporal lobe epilepsy. Epilepsia. 2012;53: 1033-43.

25. Gourmaud S, Shou H, Irwin DJ, Sansalone K, Jacobs LM, Lucas TH, et al. Alzheimer-like amyloid and tau alterations associated with cognitive deficit in temporal lobe epilepsy. Brain. 2020;143:191-209.

26. Palizvan MR, Fathollahi Y. Epileptogenic insult causes a shift in the form of long-term potentiation expression. Neuroscience. 2005;134:415-23.

27. Sommer C, Roth SU. Kainate-induced epilepsy alters protein expression of AMPA receptor subunits GluA1, GluR2 and AMPA receptor binding protein in the rat hippocampus. Acta Neuropathol. 2001;101:460-8.

28. Ma Y, Sun X, Li J, Jia RH, Yuan F, Wei D, et al. Melatonin alleviates the epilepsy-associated impairments in hippocampal LTP and spatial learning through rescue of surface GluR2 expression at hippocampal CA1 Synapses. Neurochem Res. 2017;42:1438-48,

\section{Ready to submit your research? Choose BMC and benefit from:}

- fast, convenient online submission

- thorough peer review by experienced researchers in your field

- rapid publication on acceptance

- support for research data, including large and complex data types

- gold Open Access which fosters wider collaboration and increased citations

- maximum visibility for your research: over $100 \mathrm{M}$ website views per year

At $\mathrm{BMC}$, research is always in progress.

Learn more biomedcentral.com/submissions 